TI 2011-048/4

Tinbergen Institute Discussion Paper

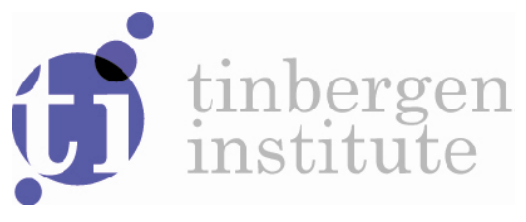

\title{
A Bayesian Analysis of Unobserved Component Models using $O x$
}

Charles S. Bos

Dept. of Econometrics and Operations Research, VU University Amsterdam, and Tinbergen Institute. 
Tinbergen Institute is the graduate school and research institute in economics of Erasmus University Rotterdam, the University of Amsterdam and VU University Amsterdam.

More TI discussion papers can be downloaded at http://www.tinbergen.nl

Tinbergen Institute has two locations:

Tinbergen Institute Amsterdam

Gustav Mahlerplein 117

1082 MS Amsterdam

The Netherlands

Tel.: +31(0)205251600

Tinbergen Institute Rotterdam

Burg. Oudlaan 50

3062 PA Rotterdam

The Netherlands

Tel.: +31(0)10 4088900

Fax: +31(0)104089031

Duisenberg school of finance is a collaboration of the Dutch financial sector and universities, with the ambition to support innovative research and offer top quality academic education in core areas of finance.

DSF research papers can be downloaded at: http://www.dsf.nl/

Duisenberg school of finance

Gustav Mahlerplein 117

1082 MS Amsterdam

The Netherlands

Tel.: +31(0)20 5258579 


\title{
A Bayesian Analysis of Unobserved Component Models using $\mathrm{Ox}$
}

\author{
Charles S. Bos* \\ Tinbergen Institute and VU University Amsterdam
}

March 2, 2011

\begin{abstract}
This article details a Bayesian analysis of the Nile river flow data, using a simple state space model. This allows the article to concentrate on implementation issues surrounding this model.

For this data set, Metropolis-Hastings and Gibbs sampling algorithms are implemented in the programming language Ox. The Markov chain Monte Carlo methods only provide output conditioned upon the full data set. For filtered output, conditioning only on past observations, the particle filter is introduced.

The sampling methods are flexible, and this advantage is used to extend the model to incorporate a stochastic volatility process. The volatility changes both in the Nile data, and for comparison also in daily S\&P 500 return data, are investigated. The posterior density of parameters and states is found to provide information on which elements of the model are easily identifiable, and which elements are estimated with less precision.
\end{abstract}

Keywords: State space methods, unobserved components, Bayes, stochastic volatility.

JEL classification: C11, C22, C52, C87.

\section{Introduction}

In this article we analyze the Nile data from a Bayesian perspective using Ox (Doornik, 2009). The initial model in Section 2 is the standard local level model. As the model is linear and Gaussian, the Kalman filter equations (see Commandeur et al., 2011) allow directly for a classical analysis. The added value of a Bayesian analysis, however, is that full information on the uncertainty of the parameters is obtained from the posterior density, and that problems with multimodality of the (likelihood or posterior) density surface are tackled more easily.

The aim of this article is not to provide the most thorough analysis of the Nile river flow data set, but instead describe the basic steps of a Bayesian analysis of the model, together with details on the implementation of the algorithms in Ox. The algorithms are chosen for the insight they give, not necessarily always for being the most advanced, quickest, or most precise option at hand. The article is complemented with a set of programs (see the listing in Appendix B) for performing the analysis.

\footnotetext{
${ }^{*}$ Corresponding author: C.S. Bos, Department of Econometrics \& O.R., VU University Amsterdam, De Boelelaan 1105, 1081 HV Amsterdam, The Netherlands. Email cbos@feweb.vu.nl, tel/fax: +31 (0)20 59860 $23 /+31(0) 205986020$.
} 
Two different sampling algorithms are presented. The Metropolis-Hastings algorithm (Section 2.2) is appropriate for the basic local level model. For illustrative purposes, a Gibbs sampling scheme is proposed in Section 2.3. Section 2.4 presents a particle filter for the model in which filtered estimates of the level component are evaluated. A filtered estimate is conditional on the past and concurrent observations while the smoothed estimate is based on all observations. The Bayesian sampling schemes from Sections 2.2 and 2.3 produce smoothed estimates. Using the estimate of the likelihood from the particle filter, the marginal likelihood is also obtained. The Gibbs sampler and particle filter are used in Section 3 to extend the local level model allowing for stochastically varying variance in the observation equation.

The algorithms are applied first on the Nile data set, with empirical results in Section 2.5. Further empirical results for the case with stochastic volatility are provided in Section 3.3, and an application on a financial data set concerning daily S\&P 500 returns follows in Section 4. Section 5 provides concluding remarks.

\section{The local level model applied to the Nile data}

\subsection{Setting up the model and prior}

In order to analyse the local level model for the Nile data using a Markov chain Monte Carlo (MCMC) setup, first an analysis of the parameters occuring in the model and their possible sampling schemes is needed. For ease of reference, the model that interests us here is

$$
\begin{aligned}
y_{t} & =\mu_{t}+\varepsilon_{t}, & & \varepsilon_{t} \sim \operatorname{NID}\left(0, \sigma_{\varepsilon}^{2}\right), \\
\mu_{t+1} & =\mu_{t}+\xi_{t}, & & \xi_{t} \sim \operatorname{NID}\left(0, \sigma_{\xi}^{2}\right),
\end{aligned}
$$

for $t=1, \ldots, T$. The model contains two parameters, $\theta=\left(\sigma_{\varepsilon}, \sigma_{\xi}\right)$, and one vector of unobserved states $\mu=\left(\mu_{1}, \ldots, \mu_{T}\right)$.

A Bayesian analysis is performed by combining prior information on the parameters with the information from the likelihood function, according to Bayes' formula

$$
\mathrm{P}\left(\theta \mid Y_{T}\right) \propto \mathcal{L}\left(Y_{T} ; \theta\right) \pi(\theta)
$$

where $\mathcal{L}\left(Y_{T} ; \theta\right)$ is the likelihood of data $Y_{T}=\left(y_{1}, \ldots, y_{T}\right)$ evaluated at the vector of parameters $\theta$, and $\pi(\theta)$ is the prior density.

As we shall see later, a convenient prior density for the standard deviations $\sigma_{\varepsilon}$ and $\sigma_{\xi}$ is the Inverted Gamma-1 (IG-1) density (see e.g. Bauwens et al., 1999),

$$
f_{\mathrm{IG}-1}(\sigma ; r, a)=\frac{2 a^{r}}{\Gamma(r) \sigma^{2 r+1}} \exp \left(-\frac{a}{\sigma^{2}}\right)
$$

with expectation and variance

$$
\mathrm{E}(\sigma)=\sqrt{a} \frac{\Gamma\left(r-\frac{1}{2}\right)}{\Gamma(r)}, \quad\left(r>\frac{1}{2}\right), \quad \operatorname{VAR}(\sigma)=\frac{a}{(r-1)}-\mathrm{E}(\sigma)^{2}, \quad(r>1) .
$$

This IG-1 density is the so-called conjugate density, implying that the posterior density of the parameters in this model is available in closed form if this specific form of prior density is chosen. 
For the present linear Gaussian model, the likelihood function $\mathcal{L}\left(Y_{T} ; \theta\right)$ can be calculated from the output of the Kalman filter (KF, see Harvey, 1989; Durbin and Koopman, 2001). Therefore, a sample from the posterior density of $\sigma_{\varepsilon}, \sigma_{\xi} \mid Y_{T}$ can be obtained using a MetropolisHastings (MH) approach (Metropolis et al., 1953; Chib and Greenberg, 1995). This will be detailed in Section 2.2. Alternatively, a Gibbs sampling approach (Smith and Gelfand, 1992) with data augmentation would consider the state $\mu$ as part of the parameter space, and successively draw from the full-conditional densities $\mathrm{P}\left(\mu \mid Y_{T}, \sigma_{\varepsilon}, \sigma_{\xi}\right), \mathrm{P}\left(\sigma_{\varepsilon} \mid Y_{T}, \mu, \sigma_{\xi}\right)$ and $\mathrm{P}\left(\sigma_{\xi} \mid Y_{T}, \mu, \sigma_{\varepsilon}\right)$. Such an approach has the advantage that also a sample from the posterior density of the states is obtained, and that it is easily extended to include elements breaking the linearity or Gaussianity of the model. This latter approach is presented in Section 2.3.

Both the $\mathrm{MH}$ and Gibbs samplers result in a posterior density of the parameters conditional on the full data set $Y_{T}$. At times, the filtered states $\mu_{t} \mid Y_{t}, \sigma_{\varepsilon}, \sigma_{\xi}$ are of interest. Section 2.4 presents a particle filter for this purpose, and is followed by Section 2.5 with empirical results on the Nile data.

\subsection{Implementing a random walk Metropolis approach}

For the Metropolis-Hastings approach, the general algorithm follows the steps:

1. Initialise, start with a vector of parameters $\theta^{(0)}$, set $i=1$;

2. Draw a candidate value $\theta^{*} \sim q\left(\theta \mid \theta^{(i-1)}\right)$;

3. Accept the new draw with probability

$$
\alpha_{\mathrm{MH}}\left(\theta^{*}, \theta^{(i-1)}\right)=\min \left[\frac{\mathrm{P}\left(\theta^{*} \mid Y_{T}\right) q\left(\theta^{(i-1)} \mid \theta^{*}\right)}{\mathrm{P}\left(\theta^{(i-1)} \mid Y_{T}\right) q\left(\theta^{*} \mid \theta^{(i-1)}\right)}, 1\right] .
$$

If the draw is accepted, set $\theta^{(i)}=\theta^{*}$, else $\theta^{(i)}=\theta^{(i-1)}$;

4. Increase $i$, and repeat from Step 2. until the sample is sufficiently large.

The candidate density $q\left(\theta \mid \theta^{(i-1)}\right)$ can be chosen freely, though a density which is related to the target density would lead to better acceptance rates. Here, a random walk Metropolis scheme is used, taking the candidate $q\left(\theta \mid \theta^{(i-1)}\right)$ to be a multivariate normal density with expectation $\theta^{(i-1)}$ and a prespecified covariance matrix,

$$
q\left(\theta \mid \theta^{(i-1)}\right) \sim \operatorname{NID}\left(\theta^{(i-1)}, \Sigma_{q}\right) .
$$

As this candidate density is symmetric, it drops from Equation (4), simplifying the sampling further.

Listing 1: The random walk Metropolis scheme in Ox

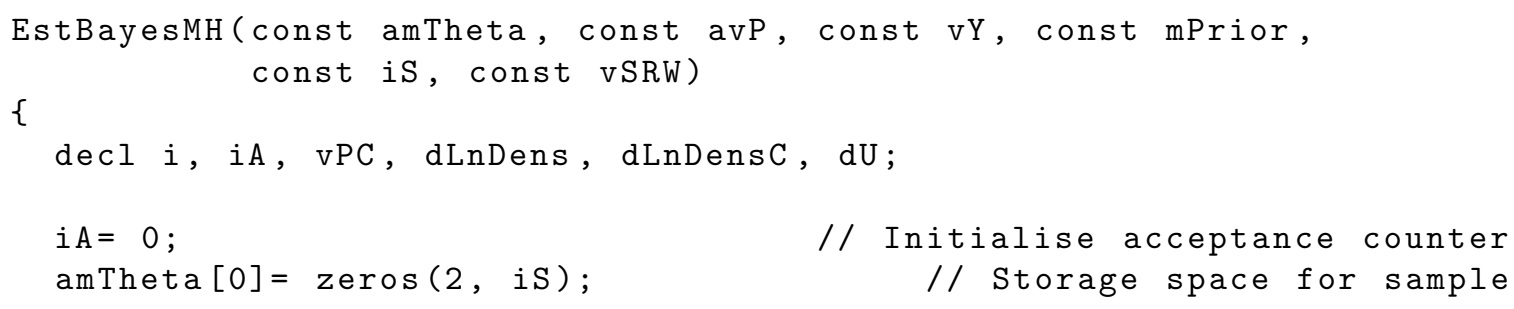




\subsection{Implementing a Gibbs approach with data augmentation}

For the second approach, the parameter space is augmented with the vector of states $\mu=$ $\left(\mu_{1}, \ldots, \mu_{T}\right)$, and successively a draw is made from the full conditional densities. The algorithm follows the steps:

1. Initialise, start with vectors $\theta^{(0)}$ and $\mu^{(0)}$, and set $i=1$;

2. Sample new states $\mu^{(i)} \sim \mathrm{P}\left(\mu \mid Y_{T}, \theta^{(i-1)}\right)$ using the simulation smoother. For a description see Appendix A or Durbin and Koopman (2002);

3. Sample $\sigma_{\varepsilon}^{(i)} \sim \mathrm{P}\left(\sigma_{\varepsilon} \mid Y_{T}, \mu^{(i)},\left[\sigma_{\xi}^{(i-1)}\right]\right)$. This density is a IG-1 density, as can be seen from

$$
\begin{aligned}
& \mathrm{P}\left(\sigma_{\varepsilon} \mid Y_{T}, \mu^{(i)},\left[\sigma_{\xi}^{(i-1)}\right]\right) \propto \mathcal{L}\left(Y_{T} ; \mu^{(i)}, \sigma_{\varepsilon}\right) \pi\left(\sigma_{\varepsilon}\right) \pi\left(\sigma_{\xi}^{(i-1)}\right) \\
& \propto \sigma_{\varepsilon}^{-T} \exp \left(-\frac{1}{2 \sigma_{\varepsilon}^{2}} \sum_{i=1}^{T}\left(y_{t}-\mu_{t}^{(i)}\right)^{2}\right) \frac{2 a_{\varepsilon}^{r_{\varepsilon}}}{\Gamma\left(r_{\varepsilon}\right) \sigma_{\varepsilon}^{2 r_{\varepsilon}+1}} \exp \left(-\frac{a_{\varepsilon}}{\sigma_{\varepsilon}^{2}}\right) \\
& \propto \frac{1}{\sigma_{\varepsilon}^{2 r_{\varepsilon}+T+1}} \exp \left(-\frac{1}{\sigma_{\varepsilon}^{2}}\left(a_{\varepsilon}+\frac{1}{2} \sum_{i=1}^{T}\left(y_{t}-\mu_{t}^{(i)}\right)^{2}\right)\right) \\
& \equiv \mathrm{IG}-1\left(r=r_{\varepsilon}+\frac{T}{2}, a=a_{\varepsilon}+\frac{1}{2} \sum\left(y_{t}-\mu_{t}^{(i)}\right)^{2}\right)
\end{aligned}
$$

4. Sample a new $\sigma_{\xi}^{(i)} \sim \mathrm{P}\left(\sigma_{\xi} \mid \mu^{(i)},\left[Y_{T}, \sigma_{\varepsilon}^{(i)}\right]\right)$. This density is again a IG-1 density, with

$$
\mathrm{P}\left(\sigma_{\xi} \mid \mu^{(i)},\left[Y_{T}, \sigma_{\varepsilon}^{(i)}\right]\right)=\mathrm{IG}-1\left(r=r_{\xi}+\frac{T-1}{2}, a=a_{\xi}+\frac{1}{2} \sum_{i=2}^{T}\left(\mu_{t}^{(i)}-\mu_{t-1}^{(i)}\right)^{2}\right)
$$

5. Increase $i$, and repeat from Step 2. until the sample is sufficiently large.

The implementation of the algorithm is nothing more than a loop, similar to Listing 1, around the three samplers introduced above. An implementation of the simulation smoother code is presented in Appendix A, with detailed explanations. For the standard deviations, a routine as in Listing 3 can draw new values from the IG-1 distribution. Notice that the Inverted Gamma-1 density relates directly to the more standard Gamma density: If $h \sim \operatorname{Gamma}(r, a)$, then $\sigma=1 / \sqrt{h} \sim \operatorname{IG}-1(r, a)$. This relationship is applied here to enable the use of the Ox function rangamma() for generating the standard deviation.

Listing 3: Simulating the standard deviations in Ox

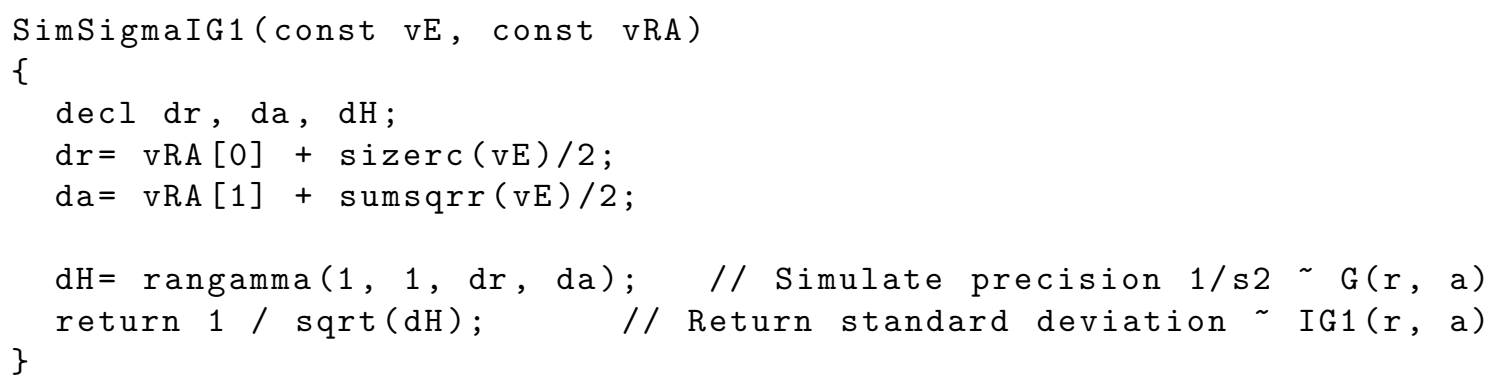


The Gibbs algorithm results in a sample from the state vector $\mu$ and parameters $\sigma_{\varepsilon}, \sigma_{\xi}$, all of them conditional on the full data set $Y_{T}=\left(y_{1}, \ldots, y_{T}\right)$. For the $\mathrm{MH}$ approach of the previous section, no posterior sample of the states was available. What however is missing for both the $\mathrm{MH}$ and Gibbs samplers, is information on the filtered quantities, like the prediction errors or the filtered state, and also the value of the likelihood. In the case of the Nile data, with the basic local level model, these quantities can be obtained from the KF. The next section describes the basic approach of particle filtering, which can deliver similar output on filtered states and the likelihood as the KF, but is not restricted to linear and Gaussian state space models.

\subsection{A particle filter}

For the fully linear unobserved components model (1)-(2), the standard Kalman filter routines can be used to extract filtered quantities. If the model is extended to include non-linear or non-Gaussian components as well, then a precise Kalman filter is no longer available, and an approximative simulation method has to be used. Cappé et al. (2007) give an overview of possible particle filter implementations. A simple version is provided here.

The main idea of the particle filter is to sample a cloud of particles, $m_{t}^{(i)}, i=1, \ldots, M$

such that they together describe the density of the state $\mu$ at time $t$ given the data up to and including $y_{t}$. The most basic particle filter is described by the following steps:

1. To initialise, simulate $m_{1}^{(i)} \mid y_{1} \sim \operatorname{NID}\left(y_{1}, \sigma_{\varepsilon}^{2}\right), i=1, \ldots, M$, where it is assumed that the prior density of the states is diffuse, such that the first observation contains all possible information on the first state. Set weights $\omega_{1}^{(i)} \equiv 1 / M$, as the particles are sampled precisely from the correct density. Set $t=2$, and commence the loop;

2. Choose $M$ particles with replacement from the current set, using weights $\omega_{t-1}^{(i)}$, and call these $\tilde{m}_{t-1}^{(j)}, j=1, \ldots, M$;

3. Propagate the particles according to a (candidate) transition density, sampling

$$
m_{t}^{(i)} \sim q\left(m_{t} \mid \tilde{m}_{t-1}^{(i)}, y_{t}\right)
$$

with corresponding weights

$$
\tilde{\omega}_{t}^{(i)}=\frac{p\left(y_{t} \mid m_{t}^{(i)}\right) p\left(m_{t}^{(i)} \mid m_{t-1}^{(i)}\right)}{M q\left(m_{t}^{(i)} \mid m_{t-1}^{(i)}, y_{t}\right)}, \quad \omega_{t}^{(i)}=\frac{\tilde{\omega}_{t}^{(i)}}{\sum_{j=1}^{M} \tilde{\omega}_{t}^{(j)}}, \quad i=1, \ldots, M
$$

4. Increase $t$, and repeat from Step 2. while $t \leq T$.

The candidate transition density $q\left(m_{t} \mid \tilde{m}_{t-1}^{(i)}, y_{t}\right)$ can make use of the observation $y_{t}$, such that a set of new particles is sampled which take the newest observation into account. A simple choice, however, is to take

$$
q\left(m_{t} \mid \tilde{m}_{t-1}^{(i)}, y_{t}\right) \equiv p\left(m_{t} \mid \tilde{m}_{t-1}^{(i)}\right) \sim \operatorname{NID}\left(m_{t-1}^{(i)}, \sigma_{\xi}^{2}\right) .
$$

In the latter case, the weights in Step 3. simplify, and only contain the likelihood contributions. The alternative is to implement a single step of the filtering equation of the Kalman 
filter, and sample from the resulting density. This would lead to a better set of particles, with more uniformly distributed weights, at the cost of a slight increase in difficulty of implementation. In Bos and Shephard (2006) the particles even consist of the first two moments of the states, updated using the Kalman equations, and hence an exact filter results for a linear and Gaussian unobserved components model.

In case a certain observation is not available, the weights can obviously not be calculated. In such a situation, equal weights are set, and the algorithm continues. This is the way that a forecast from the state can be made, straight from the particle filter.

Listing 4: The particle filter in Ox

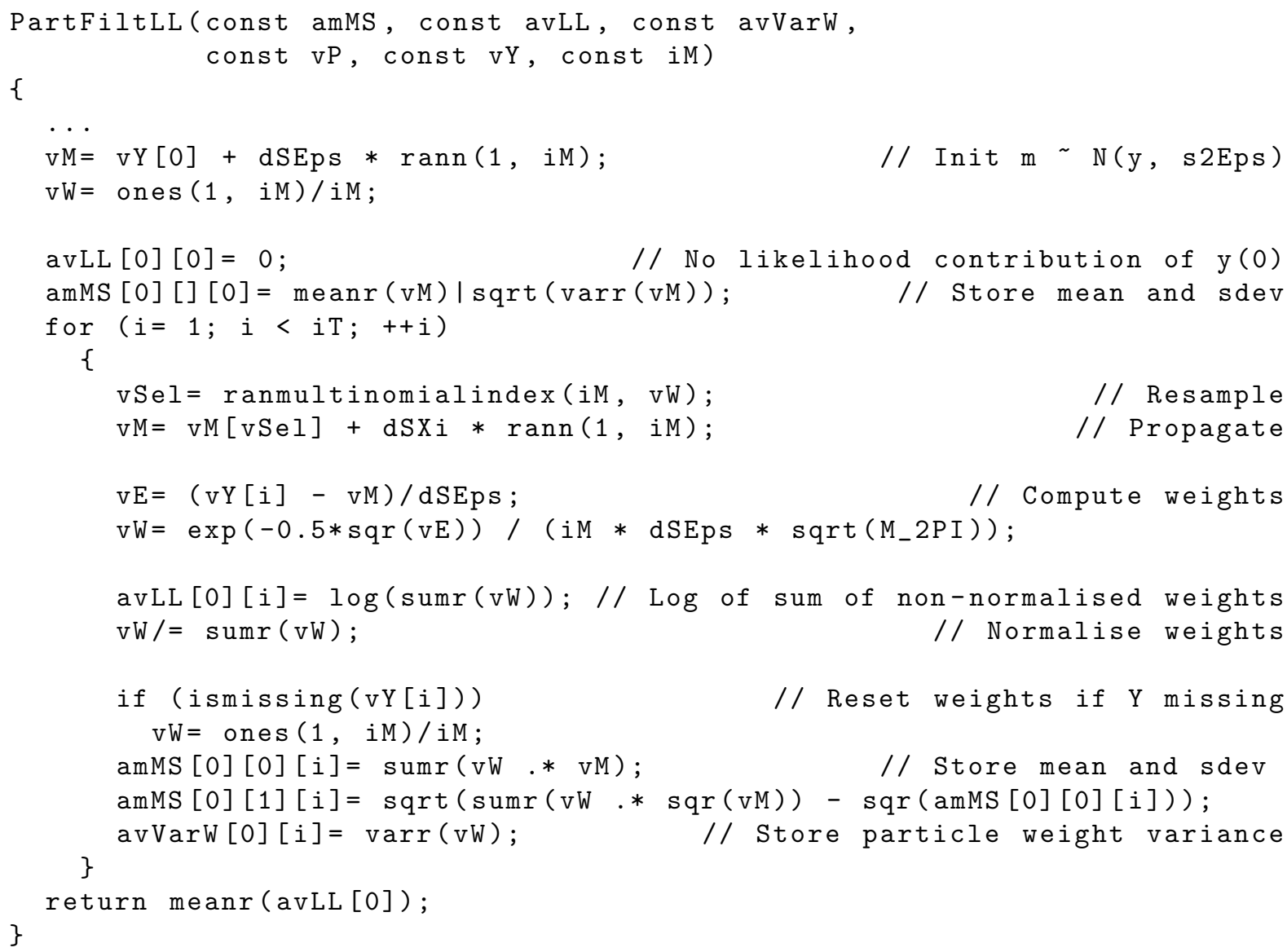

From the particle filter, output on the filtered particles can be collected. A basic implementation of the particle filter is provided in Listing 4 . The mean and standard deviation of the particles is collected during the filter, and based on the propagated particles before resampling using weights $\omega_{t}$ (e.g. $\bar{m}_{t}=\sum_{i} \omega_{t}^{(i)} m_{t}^{(i)}$ ). Alternatively, the mean and standard deviation could be constructed from the particles after resampling (i.e., $\bar{m}_{t}=\frac{1}{M} \sum_{i} \tilde{m}_{t}^{(i)}$ ), but this latter option induces a loss of efficiency due to the resampling.

One of the outputs of the above particle filter is the loglikelihood contribution of each observation, which equals the logarithm of the sum of non-normalised weights (see Pitt and Shephard, 1999). The sum of the loglikelihood contributions delivers the loglikelihood of the data for a given set of parameters, integrating out the unobserved states. The likelihood $\mathcal{L}\left(Y_{T} ; \theta\right)$ in turn can be used as input for computing the marginal likelihood $M\left(Y_{T} \mid \mathcal{M}\right)$ of a model $\mathcal{M}$, which is the likelihood of the model integrating out the parameters with respect 
to the prior $\pi(\theta)$ :

$$
M\left(Y_{T} \mid \mathcal{M}\right)=\int_{\theta} \mathcal{L}\left(Y_{T} ; \theta\right) \pi(\theta) \partial \theta \equiv \frac{\mathcal{L}\left(Y_{T} ; \theta\right) \pi(\theta)}{\mathrm{P}\left(\theta \mid Y_{T}\right)} .
$$

In this formula, the value of the posterior density $\mathrm{P}\left(\theta \mid Y_{T}\right)$ needs to be computed including the integrating constant. Several methods exist, but a simple one uses a Laplace approximation for $\mathrm{P}\left(\theta \mid Y_{T}\right)$ at the posterior mode. Evaluating this approximation at the posterior mode itself gives $\mathrm{P}\left(\tilde{\theta} \mid Y_{T}\right) \approx(2 \pi)^{-k / 2}\left|\Sigma_{\theta}\right|^{-1 / 2} \exp \left(-\frac{1}{2} \times(\tilde{\theta}-\tilde{\theta})\right)=(2 \pi)^{-k / 2}\left|\Sigma_{\theta}\right|^{-1 / 2}$, with $k$ the dimension of the parameter vector and $\Sigma_{\theta}$ the posterior covariance, see also Listing 5 . For small dimensions of the parameter vector and near-Gaussian posterior densities, this Laplace approximation of the marginal likelihood can be sufficiently precise (Kass et al., 1990; Bos, 2002).

Listing 5: Marginal loglikelihood using Laplace approximation in Ox

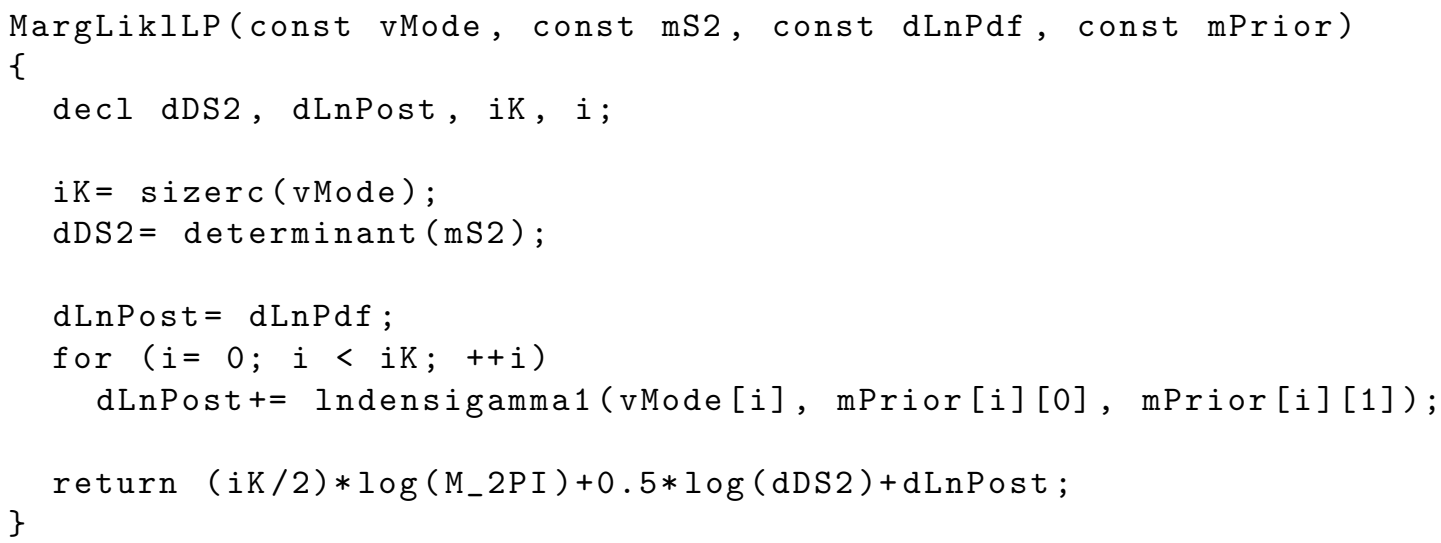

The Bayes factor $B_{12}$ (Jeffreys, 1961) is calculated using the marginal likelihood from two competing models $\mathcal{M}_{1}$ and $\mathcal{M}_{2}$, as in

$$
B_{12}=\frac{M\left(Y \mid \mathcal{M}_{1}\right)}{M\left(Y \mid \mathcal{M}_{2}\right)}
$$

This odds ratio is the factor by which the data considers $\mathcal{M}_{1}$ more probable than the alternative $\mathcal{M}_{2}$. Kass and Raftery (1995) consider twice the difference in log-marginal likelihoods, and describe that a difference of $2<2 B_{12}<6$ gives 'positive evidence' for model 1, and $2 B_{12}>10$ gives 'very strong evidence'. These marginal likelihoods and their differences will be used in Section 3 and later to decide whether there is evidence in the data for the inclusion of a stochastic volatility effect in the model.

\subsection{Empirical results on samplers for Nile river flow}

Both the Metropolis and the Gibbs sampler depend on the specification of the prior density for the parameters. The conjugate IG-1 density was chosen for the two standard deviations in the model. Table 1 provides the starting values $\theta_{0}$, the parameters of the IG- 1 prior density, and the corresponding prior mean $\bar{\theta}$ and standard deviation $\sigma_{\theta}$. The priors are chosen such that their expectation is somewhat in the neighbourhood of the classical estimates, with standard deviations which allow for a considerable amount of uncertainty. For the Metropolis sampler, the random walk standard deviation is taken to be one tenth of the prior standard deviation. 
With these values, the two samplers were run, both first for 10,000 iterations to allow the sampler to burn-in, then for 100,000 further iterations to collect the final sample. This was found to be a sufficiently large sample to obtain convergence. The posterior mean $\bar{\theta}$, standard deviation $\sigma_{\theta}$, and inefficiency measure $R_{B}$ of the parameters are displayed in Table 2 , together with maximum likelihood estimates $\hat{\theta}, \sigma_{\theta}$ of the parameters in the first two columns. Figure 1 plots the posterior densities together with the prior densities and the autocorrelations of the sample.

Table 1: Starting values and prior density for parameters Nile data

\begin{tabular}{rrrrrr} 
& $\theta_{0}$ & $r$ & $a$ & $\bar{\theta}$ & $\sigma_{\theta}$ \\
\hline$\sigma_{\varepsilon}$ & 120 & 2.66 & 30,000 & 125 & $(50)$ \\
$\sigma_{\xi}$ & 30 & 2 & 5,000 & 63 & $(33)$ \\
\hline \hline
\end{tabular}

Table 2: Estimation results of local level model on Nile data

\begin{tabular}{rcrrrrrrr}
\multicolumn{1}{c}{ ML } & \multicolumn{3}{c}{ MH } & \multicolumn{3}{c}{ Gibbs } \\
& $\hat{\theta}$ & $\sigma_{\theta}$ & $\bar{\theta}$ & $\sigma_{\theta}$ & $R_{B}$ & $\bar{\theta}$ & $\sigma_{\theta}$ & $R_{B}$ \\
\hline$\sigma_{\varepsilon}$ & 122.876 & $(12.81)$ & 118.799 & $(10.90)$ & {$[57.7]$} & 118.694 & $(11.10)$ & {$[4.5]$} \\
$\sigma_{\xi}$ & 38.332 & $(16.72)$ & 47.665 & $(11.31)$ & {$[90.5]$} & 48.011 & $(11.65)$ & {$[12.9]$} \\
Loglikelihood KF & -632.546 & & & & & & \\
Loglikelihood PF & -632.669 & & -632.723 & & -632.639 & \\
Acceptance rate & & 0.792 & & & \\
Duration & $0.53 \mathrm{~s}$ & & $2.66 \mathrm{~s}$ & & $4.75 \mathrm{~s}$ & \\
\hline \hline
\end{tabular}
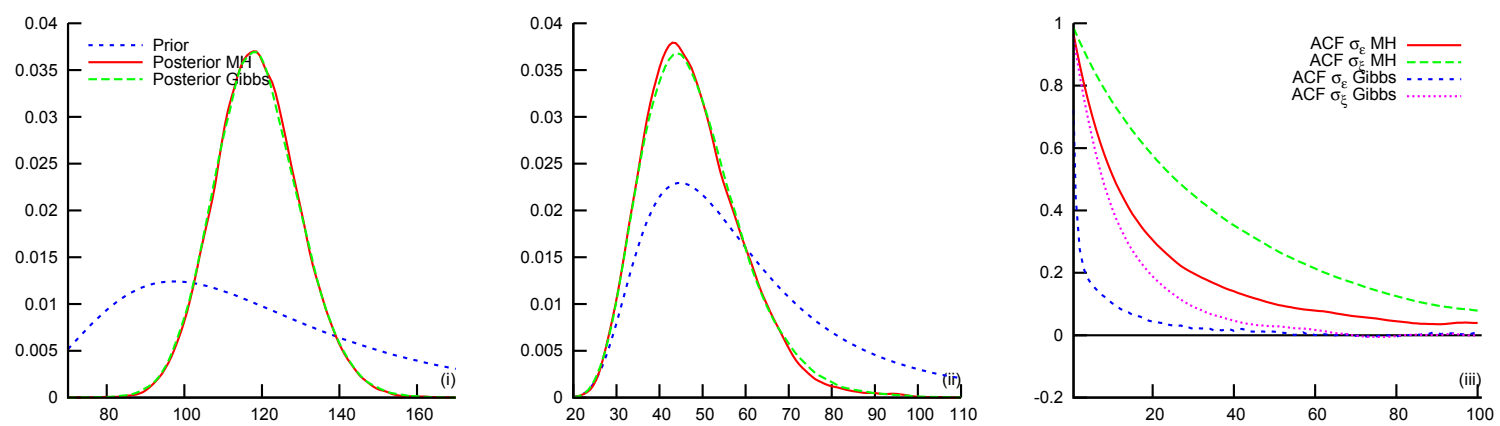

Figure 1: Prior and posterior densities for $\sigma_{\varepsilon}$ (panel (i)) and $\sigma_{\xi}$ (panel (ii)) for the Nile data, with autocorrelations in panel (iii), comparing $\mathrm{MH}$ and Gibbs results

The Nile data is found to be little informative on the precise location of the parameters, with large standard deviations for $\sigma_{\varepsilon}$ and $\sigma_{\xi}$. Figure 1 shows that, especially for the state transition standard deviation $\sigma_{\xi}$, the prior influences the location of the posterior. The posterior densities of the $\mathrm{MH}$ and Gibbs samplers are similarly shaped, though the autocorrelation functions of the sample show how the Gibbs chain in this case delivers a sample with lower autocorrelation between successive draws. This lower autocorrelation also is apparent from the values for the inefficiency measures $R_{B}$ in columns 5 and 7 of Table 2 . These inefficiency measures display the relative variance of the posterior sample draws when adapting for the 

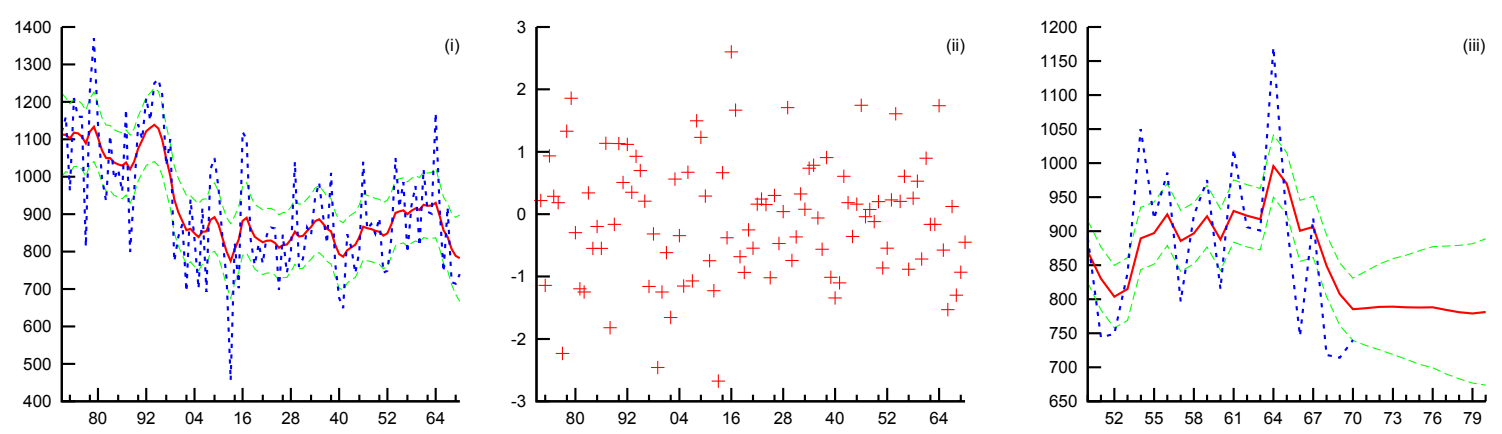

Figure 2: Nile data, with smoothed state and 90\% confidence interval (panel (i)), standardised prediction errors (panel (ii)), and filtered state plus forecast with $50 \%$ confidence interval (panel (iii))

correlation between iterations, as compared to the variance without accounting for correlation. In these calculations, a bandwidth $B$ of $10 \%$ of the sample size is used. See Kim et al. (1998) or Geweke (1992) for a further background on these measures.

Table 2 also reports the loglikelihood as it is calculated through the Kalman filter, and the value that results from the particle filter of Section 2.4, using 10,000 particles. The likelihood of the particle filter is virtually equal to the one computed using the Kalman filter, with the difference due to remaining sampling variation.

The fifth row displays the acceptance rate of the random walk Metropolis candidate drawings, which is $80 \%$. The duration of the programs (on a $2.67 \mathrm{Ghz}$ Xeon X5550 processor) indicates that for such a small data set, running time is not a problem.

Figure 2 displays the Nile data together with the smoothed state estimate and the $90 \%$ confidence interval of the state in the first panel; this graph should be directly comparable to the smoothed estimates in other articles in the present special issue. The standardised prediction errors in the second panel stem from output of the particle filter: Using the filtered state, a further prediction step is taken, and the standardised prediction errors are extracted. The last panel displays the filtered state since 1950, extending the sample until 1980 to provide a forecast with a $50 \%$ confidence bound.

\section{Introducing time varying volatility}

\subsection{Extending the model}

The unobserved components model for the Nile data as described above is a linear Gaussian state space model. This simplifies the analysis considerably, as the Kalman filtering equations can be used directly. One could however wonder whether e.g. the volatility of the annual flow volume of the Nile is constant over time. As the flow will depend on matters like population, water use for irrigation, or the construction of dams in the river, one could expect changes in the process of the flow series.

Note that looking back at e.g. Figure 2, where the data itself was plotted, does not give a clear indication of changes in volatility. Besides, the number of observations $(T=100)$ is relatively low for detecting variance changes. Hence this second case will serve as a preparation for the last application, where volatility in financial returns is studied. 
To introduce a simple variant of the local level model, with a second unobserved state for the stochastic volatility, the observation equation (1) is adapted to

$$
y_{t}=\mu_{t}+\varepsilon_{t}, \quad \varepsilon_{t} \sim \operatorname{NID}\left(0, \sigma_{\varepsilon}^{2} \exp \left(h_{t}\right)\right) .
$$

A second state equation is added, describing the evolution of the (log) volatility $h_{t}$ as

$$
h_{t+1}=h_{t}+\nu_{t} \quad \nu_{t} \sim \operatorname{NID}\left(0, \sigma_{\nu}^{2}\right),
$$

initialising $h_{1} \equiv 0$. With this specification, the initial variance of the observation equation is set to $\sigma_{\varepsilon}^{2}$, whereas at later time periods the log-variance can change according to a random walk.

\subsection{Implementing the extended sampler}

Using the Gibbs approach with data augmentation, we have a parameter vector consisting of $\theta=\left(\sigma_{\varepsilon}, \sigma_{\xi}, \sigma_{\nu}\right)$ together with state variables $\mu$ and $h$. Conditional on $h$ and $\sigma_{\nu}$, the previous Gibbs sampler works well, as the unobserved components model is still conditionally linear and Gaussian. The algorithm however needs two additional steps, one for simulating $h \mid Y_{T}, \mu, \theta$ and one for $\sigma_{\nu} \mid Y_{T}, \mu, h, \sigma_{\varepsilon}, \sigma_{\xi}$. The adapted sampling scheme becomes:

1. Initialise, start with a drawing $\theta^{(0)}, \mu^{(0)}$ and $h^{(0)}$, and set $i=1$;

2. Sample $\mu^{(i)} \sim \mathrm{P}\left(\mu \mid Y_{T}, h^{(i)}, \theta^{(i)}\right)$ using the simulation smoother, taking the time varying variances into account;

3. For $t=2, \ldots, T$, the single-site volatility sampler draws successively

$$
h_{t}^{(i)} \sim \mathrm{P}\left(h_{t} \mid h_{t-1}^{(i)}, h_{t+1}^{(i-1)}, y_{t}, \mu_{t}^{(i)}\right)
$$

using an acceptance-rejection scheme (Kim et al., 1998). This is performed by sampling a candidate $h_{t}^{c}$ using

$$
\begin{array}{rlr}
h_{t}^{c} & \sim \mathrm{NID}\left(h_{t}^{*}+\frac{1}{2} v^{2}\left(\left(y_{t}-\mu^{(i)}\right)^{2} \exp \left(-h_{t}^{*}\right)-1\right), v^{2}\right), \\
h_{t}^{*} \equiv \frac{1}{2}\left(h_{t-1}^{(i)}+h_{t+1}^{(i-1)}\right), & v^{2}=\frac{1}{2} \sigma_{\nu}^{2} .
\end{array}
$$

Such a candidate draw is accepted with probability

$$
\alpha\left(h_{t}^{c}\right)=\frac{\exp \left(-\frac{1}{2}\left(y_{t}-\mu_{t}^{(i)}\right)^{2} \exp \left(-h_{t}^{c}\right)\right)}{\exp \left(-\frac{1}{2}\left(y_{t}-\mu_{t}^{(i)}\right)^{2}\left(\exp \left(h_{t}^{*}\right)\left(1+h_{t}^{*}\right)-h_{t}^{c} \exp \left(-h_{t}^{*}\right)\right)\right.} .
$$

If the candidate $h_{t}^{c}$ is accepted, set $h_{t}^{(i)}=h_{t}^{c}$, else continue drawing a new candidate $h_{t}^{c}$. Note that when $t=T$, there is no $h_{t+1}$ available, and hence $h_{t}^{*}$ and $v^{2}$ have to be adapted slightly;

4. Sample $\sigma_{\varepsilon}^{(i)} \sim \mathrm{IG}-1\left(r=r_{\varepsilon}+\frac{T}{2}, a=a_{\varepsilon}+\frac{1}{2} \sum \frac{\left(y_{t}-\mu_{t}^{(i)}\right)^{2}}{\exp \left(h_{t}^{(i)}\right)}\right)$; 
5. Sample $\sigma_{\xi}^{(i)} \sim$ IG-1 $\left(r=r_{\xi}+\frac{T-1}{2}, a=a_{\xi}+\frac{1}{2} \sum_{i=2}^{T}\left(\mu_{t}^{(i)}-\mu_{t-1}^{(i)}\right)^{2}\right)$;

6. Sample $\sigma_{\nu}^{(i)} \sim \operatorname{IG}-1\left(r=r_{\nu}+\frac{T-1}{2}, a=a_{\nu}+\frac{1}{2} \sum_{i=2}^{T}\left(h_{t}^{(i)}-h_{t-1}^{(i)}\right)^{2}\right)$;

7. Increase $i$, and repeat from Step 2. until the sample is sufficiently large.

Implementing Step 3 is relatively straightforward. Listing 6 gives an example implementation of this single-site volatility sampler for $h_{t}$. Notice however that the present algorithm now contains an outer loop to sample a large collection of parameters, and within an inner loop over $t$ it samples $h$. This implies that the code within the single-site sampler is executed very often. The routine in Listing 6 would be a first candidate for moving into a lower-level language like $\mathrm{C}$, in order to decrease the running time of the algorithm considerably. This however lies beyond the scope of the present article.

Listing 6: The single-site volatility sampler for $h_{t} \mid h_{t-1}, h_{t+1}, e_{t}$ in Ox

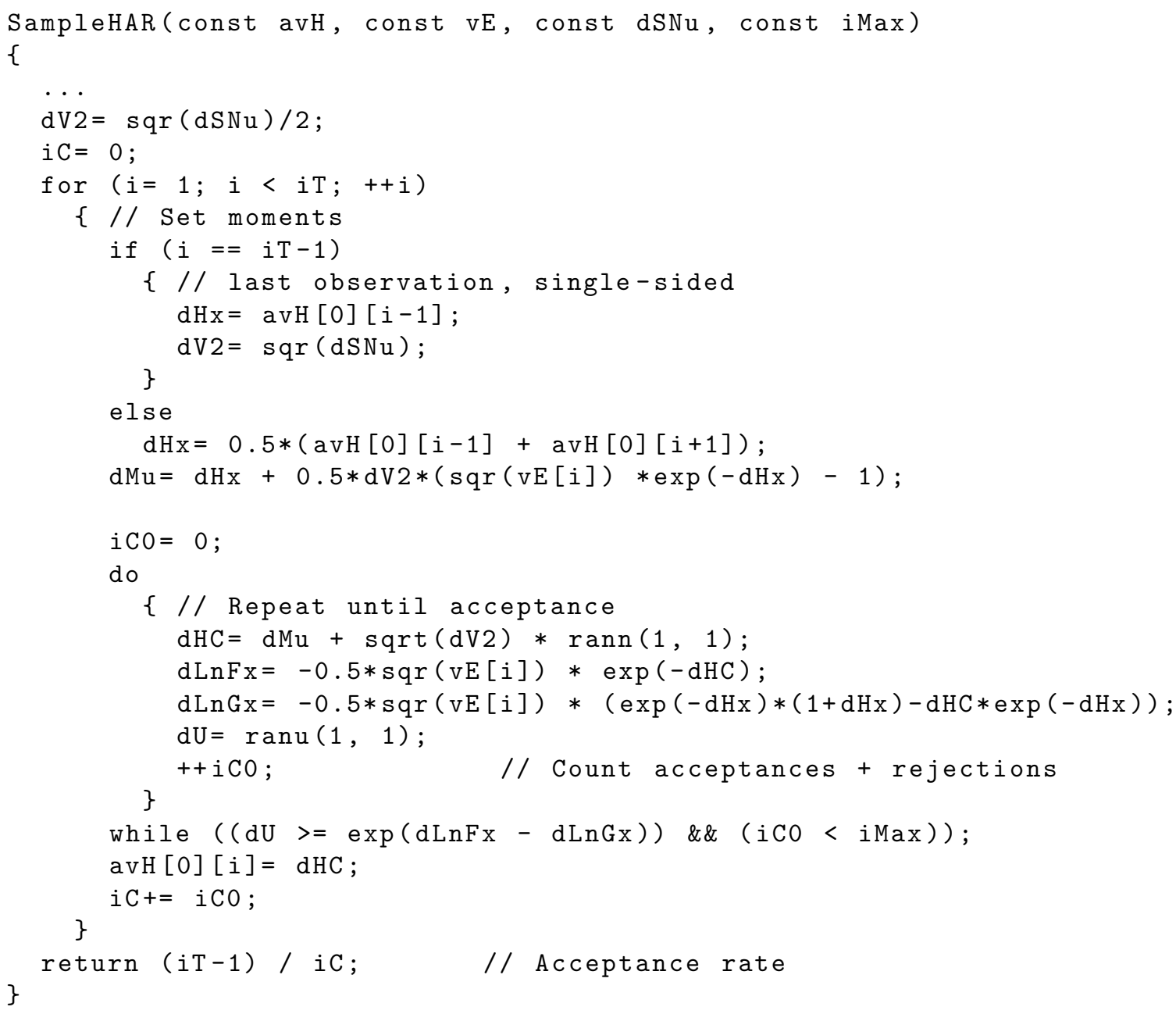

Listing 6 literally follows the proposed algorithm for sampling $h_{t} \mid h_{t-1}, h_{t+1}, y_{t}, \theta$. New candidate values $h_{t}^{c}$ should be drawn until acceptance occurs. In some cases (especially for an unlucky choice of starting values) the probability of acceptance can become very low, and it is better to force acceptance after a limited number of trials. Here, the sampling continues 
until at most a total of iMax candidates have been drawn. In well-specified situations, the acceptance rate tends to be above $95 \%$, and hence the do loop does not tend to reach a forced end in normal situations.

\subsection{Empirical results on the volatility of Nile data}

Some output of the sampler is provided in Figures 3-4. Again, a sample of 100,000 iterations was collected, after a burn-in of 10,000 iterations. The same priors as in Table 1 were used, with additionally $\pi\left(\sigma_{\nu}\right)=\mathrm{IG}-1(r=2, a=0.015)$, implying that $E\left(\sigma_{\nu}\right) \approx 0.1$ with prior standard deviation $\sigma\left(\sigma_{\nu}\right) \approx 0.05$.
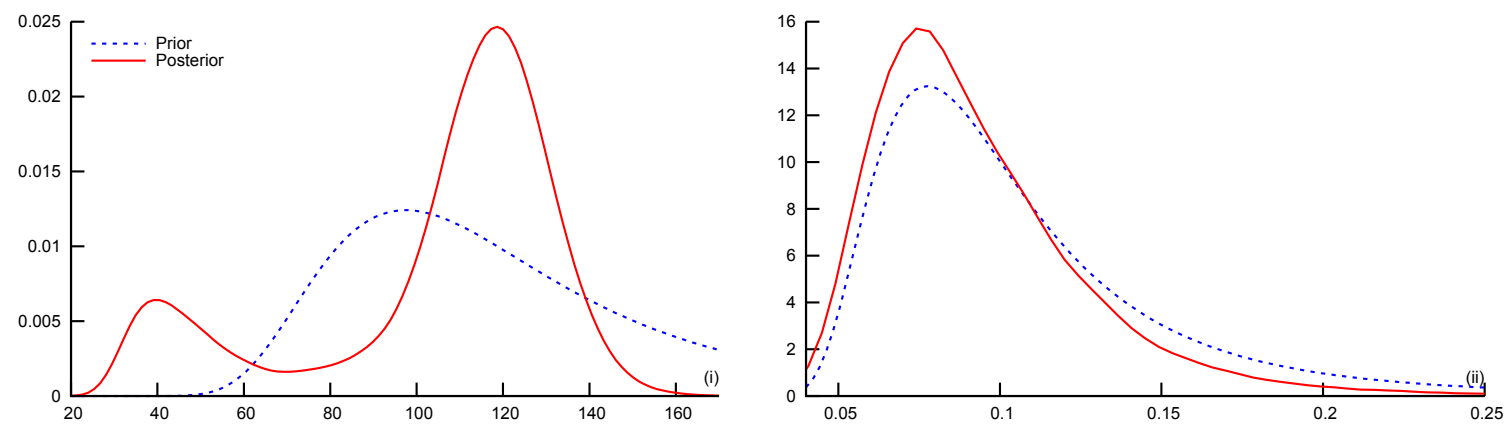

Figure 3: Prior and posterior density of $\sigma_{\varepsilon}$ (panel (i)), and $\sigma_{\nu}$ (panel (ii))
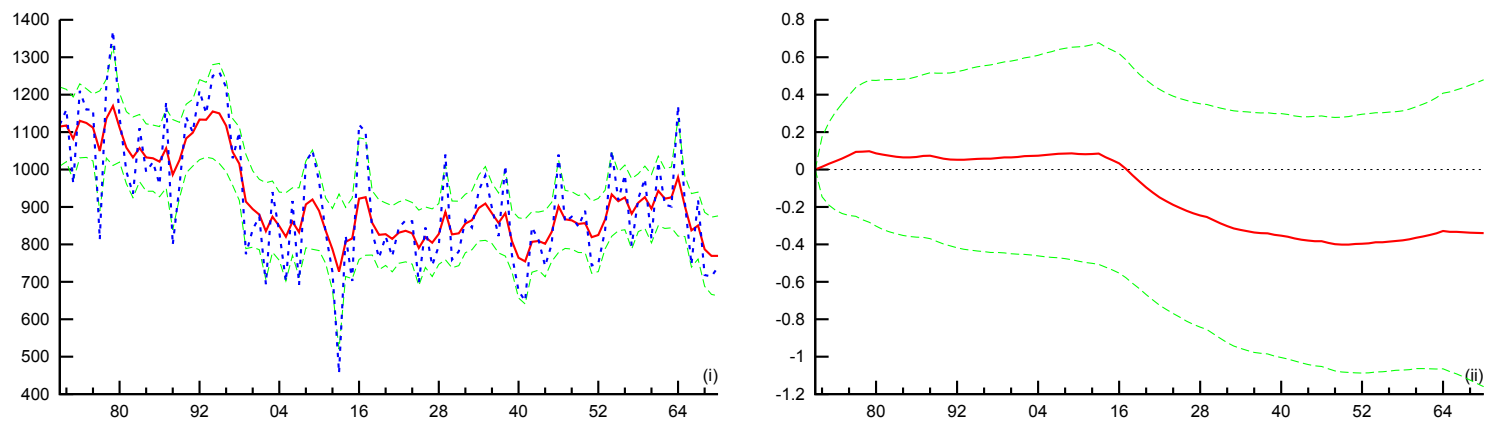

Figure 4: Nile data with smoothed level states and 90\% confidence interval (panel (i)), and smoothed volatility with confidence interval (panel (ii))

The posterior density of $\sigma_{\varepsilon}$ indicates already that this extensive time varying volatility model might be too much to estimate from this small data set. The posterior density is bimodal, implying that apart from the solution with $\sigma_{\varepsilon} \approx 120$ an alternative parameter setting with $\sigma_{\varepsilon} \approx 40$ (and $\sigma_{\xi} \approx 150$, not displayed) could work: In that case, the state effectively follows the observations very closely, with the observation equation allowing extra variability for the outliers. In panel (ii) the standard deviation $\sigma_{\nu}$ of the stochastic volatility increments is depicted. As it was already hard to estimate a constant level of volatility, estimating volatility of volatility is too much using a data set of 100 observations, and the posterior closely follows the prior density.

Table 3 displays the posterior mode, the standard deviation and the inefficiency measure, both for the model without stochastic volatility of the previous section and for the model 
Table 3: Estimation results comparing local level model without/with stochastic volatility, on Nile data

\begin{tabular}{rrrrrrr} 
& \multicolumn{3}{c}{ LL } & \multicolumn{4}{c}{ LL-SV } \\
& $\tilde{\theta}$ & $\sigma_{\theta}$ & \multicolumn{1}{c}{$R_{B}$} & \multicolumn{1}{c}{$\tilde{c}$} & $\sigma_{\theta}$ & \multicolumn{1}{c}{$R_{B}$} \\
\hline$\sigma_{\varepsilon}$ & 121.079 & $(11.10)$ & {$[4.5]$} & 116.027 & $(29.88)$ & {$[1266.9]$} \\
$\sigma_{\xi}$ & 45.392 & $(11.65)$ & {$[12.9]$} & 41.522 & $(39.53)$ & {$[1263.8]$} \\
$\sigma_{\nu}$ & & & & 0.073 & $(0.04)$ & {$[340.2]$} \\
Marginal loglikelihood & & -634.47 & & & -633.77 & \\
Duration & & $4.25 \mathrm{~s}$ & & & $29.16 \mathrm{~s}$ & \\
\hline \hline
\end{tabular}

with time varying variance. The common parameters change little, though the uncertainty on the parameters increases strongly. The large inefficiency measures for the LL-SV model indicate that correlation in the chain is high, and effectively imply that the sampler did not converge; cf. the bimodal posterior distribution for $\sigma_{\varepsilon}$.

The standard deviation $\sigma_{\nu}$ of the volatility equation is small, which is consistent with a smooth volatility process. The row with the duration of the programs indicates that sampling the volatility process is relatively costly, as a separate sampling step for each $h_{t} \mid h_{t-1}, h_{t+1}, y_{t}, \theta$ has to be performed. The table includes a row reporting the marginal loglikelihood (see Section 2.4). In this case there is only a minor difference between the two marginal loglikelihoods, implying that the model with stochastic volatility does not fit the data much better than the model with fixed volatility.

Figure 4 displays the smoothed level of the Nile data, in panel (i), and the extracted volatility process, in panel (ii), both with $90 \%$ confidence intervals. Volatility appears constant until around 1914, moving to a slightly lower level afterwards. Uncertainty about the volatility is large, as was to be expected. It is unclear what exactly causes the drop in volatility. One possible explanation is the building and extension of the Aswan dam. In 1912 an improvement to the dam was made, which could have led to a better regulation of the river, leading to lower volatility in the flows. The change in volatility however is minor, consistent with the finding that the marginal likelihoods of the models with/without SV are virtually the same.

This section illustrates the relative ease with which the standard linear Gaussian unobserved components model is extended to the case with stochastic volatility. Conditional on the volatility, the sampler hardly changes; the only real change in the algorithm is the inclusion of a sampler for the volatilities, and for the parameter $\sigma_{\nu}$ in the volatility process.

Note that the present single-site volatility sampler is a relatively simple sampler. It may lead to extensive correlation in the sampling chain as each of the $T-1$ volatilities $h_{t}$ is sampled successively, conditionally on past and future values $h_{t-1}, h_{t+1}$. Alternative approaches, like the mixture sampler of Kim et al. (1998), could lower correlation between the draws in the Markov chain.

\section{Time varying volatility for S\&P 500 returns}

\subsection{Introducing S\&P 500 data}

Figure 5 displays the S\&P 500 index data (source: Yahoo finance) for the period 2000/1/32009/12/30. The first panel displays the level of the series, followed by the returns in panel (ii). From this second panel the strong differences in volatility are immediately apparent. 
The third panel plots the average return and the average standard deviation of the S\&P 500 series, using a running window of one year. For the variance, the tranquil period between 2004-2008 is clear, with a strong increase in variance over the last two years of the sample. The average yearly return depicts roughly the opposite movement: When volatility is low, average returns were higher, and vice versa especially in the period after 2008 . The question is whether a local level with stochastic volatility model on the returns of the S\&P 500 would be able to recover these two movements. Notice that in this case the volatility is the clear signal, as that is easily detected from the movements in panel (ii). The differing average returns are not apparent from the middle panel, and hence will be harder to estimate.
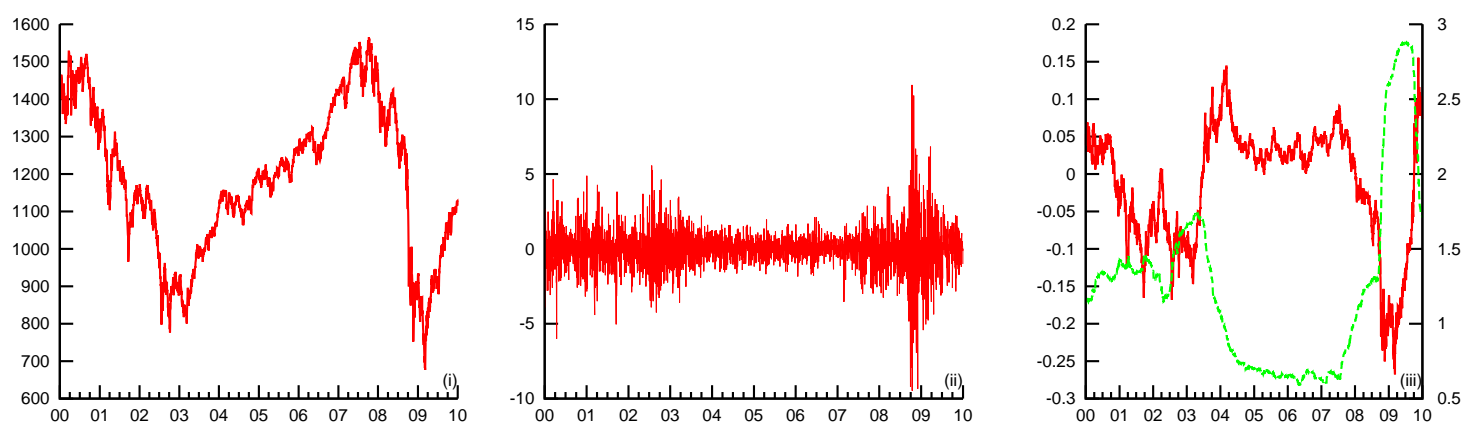

Figure 5: S\&P 500 data over 2000-2009 in levels (panel (i)), returns (ii), and the yearly average return and standard deviation (iii)

\subsection{Volatility estimation on S\&P 500 returns}

The sampler from the previous section works without change for the present data set. The only change needed in the program is the specification of the prior and the starting values of the parameters. Table 4 displays these specifications for the S\&P 500 data, cf. Table 1.

Table 4: Starting values and prior density for parameters S\&P500 data

\begin{tabular}{rrrrrr} 
& $\theta_{0}$ & $r$ & $a$ & $\bar{\theta}$ & $\sigma_{\theta}$ \\
\hline$\sigma_{\varepsilon}$ & 1 & 2 & 1.5 & 1.1 & 0.57 \\
$\sigma_{\xi}$ & 0.001 & 2 & 0.00015 & 0.011 & 0.0057 \\
$\sigma_{\nu}$ & 0.1 & 2 & 0.015 & 0.11 & 0.057 \\
\hline \hline
\end{tabular}

Results on the posterior sample are gathered in Table 5, again comparing the local level model with the model including stochastic volatility. In both cases, a Gibbs sampler of 10,000 burn-in iterations, with a final sample size of 100,000 parameter vectors is run, followed by a particle filter with 10,000 particles as before. Reported in the table are the posterior mode $\tilde{\theta}$, the posterior standard deviation $\sigma_{\theta}$, and the inefficiency measure $R_{B}$ at a bandwidth of $10 \%$ of the sample.

We first discuss the estimation results of the parameters. For the common parameters $\sigma_{\varepsilon}$ and $\sigma_{\xi}$, inclusion of the SV sequence makes little difference in the estimated values. The chain delivers strongly correlated drawings from $\sigma_{\xi}, \sigma_{\nu}$, as indicated by the high inefficiency values for these parameters. Estimation using the Gibbs sampler took 8.5 times as long when the stochastic volatility sequence was introduced: Sampling volatility is computationally hard. 
Table 5: Estimation results for S\&P 500 data

\begin{tabular}{rrrrrrrr} 
& $\tilde{\theta}$ & $\sigma_{\theta}$ & $R_{B}$ & $\tilde{\theta}$ & $\sigma_{\theta}$ & $R_{B}$ \\
\hline$\sigma_{\varepsilon}$ & 1.39994 & $(0.0197)$ & {$[0.5]$} & 1.39755 & $(0.0198)$ & {$[0.0]$} \\
$\sigma_{\xi}$ & 0.00622 & $(0.0018)$ & {$[399.5]$} & 0.00544 & $(0.0013)$ & {$[390.2]$} \\
$\sigma_{\nu}$ & & & & 0.10866 & $(0.0120)$ & {$[882.5]$} \\
Marginal loglikelihood & & -4424.44 & & & -3783.28 & \\
Duration (Gibbs/PF) & \multicolumn{3}{c}{$1 \mathrm{~m} 29 \mathrm{~s} / 13 \mathrm{~s}$} & \multicolumn{3}{c}{$12 \mathrm{~m} 41 \mathrm{~s} / 15 \mathrm{~s}$} \\
\hline \hline
\end{tabular}

Figure 6 displays the prior and posterior densities of these parameters, together with the autocorrelation functions, effectively giving the same information as the table. Here it is even clearer that the transition standard deviation, in panel (ii), is hard to estimate, with the posterior pushing downwards in comparison with the prior. The prior is informative in this situation, as it forces the posterior away from zero.
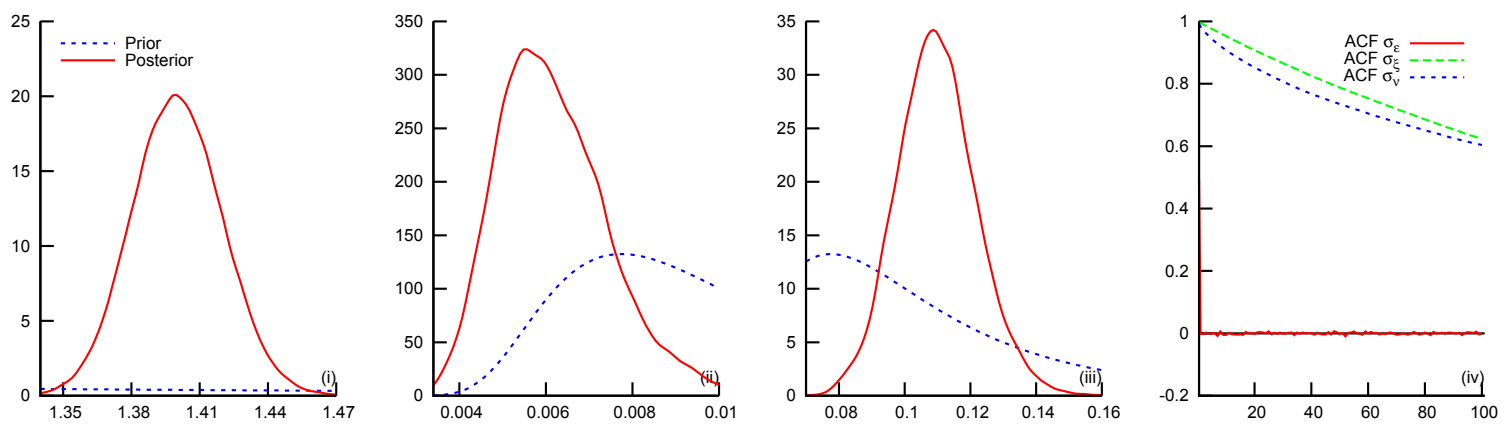

Figure 6: Prior and posterior density for $\sigma_{\varepsilon}$ (panel (i)), $\sigma_{\xi}$ (ii), $\sigma_{\nu}$ (iii) and the autocorrelations of the parameters (iv), for S\&P 500 data
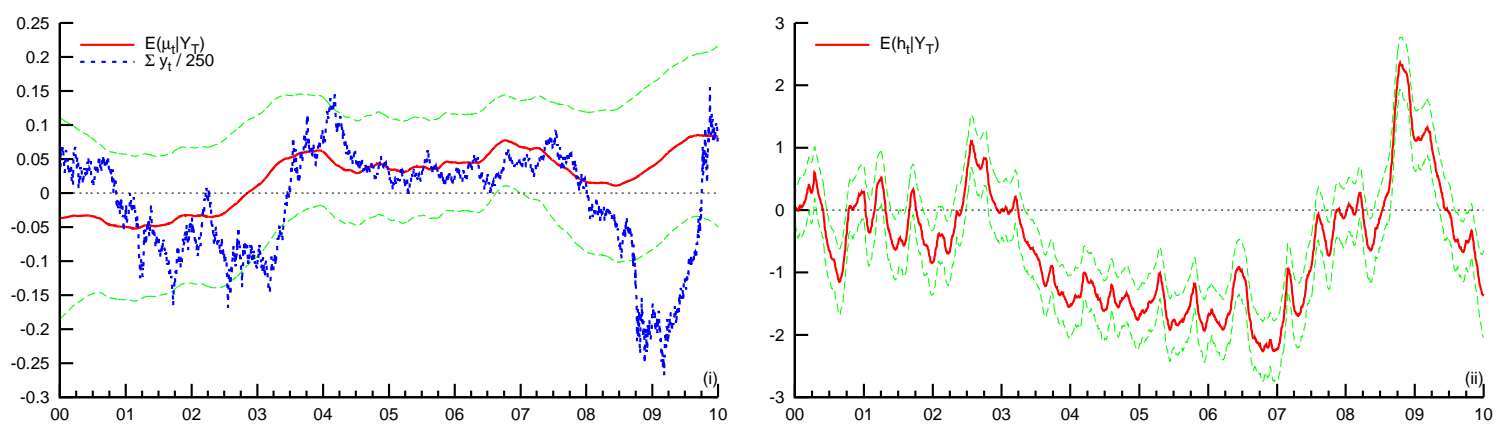

Figure 7: S\&P 500 smoothed states, for the level (panel (i)), with 90\% confidence bounds and with the average yearly return superimposed, and for the volatility (panel (ii))

The standard deviation $\sigma_{\xi}$ determines the smoothness of the state variable $\mu_{t}$ in the model. Panel (ii) in Figure 7 displays the smoothed state estimate, which indeeds indicates what periods tended to display positive or negative returns. The present estimate however is far smoother than the running yearly average return. The $90 \%$ confidence bounds include the 
value of zero over the full sample, indicating that at no period in time the expected return was clearly positive or negative.

A different prior specification for $\sigma_{\xi}$ could force the level to be more or less smooth, by putting more prior weight on smaller or larger values of $\sigma_{\xi}$ than the present choice of prior. Depending on the purpose of extracting the expected returns, the flexibility of the Bayesian approach can be an advantage in this respect, extracting a smoother or more volatile signal.

For the S\&P 500 returns, the volatility is clearly changing over time. The period 20042008 is found to display low variance, although the uncertainty slowly starts to rise in the second half of 2007. According to these estimates, the peak in uncertainty was reached in the beginning of 2009, with volatility retreating towards levels comparable to the years 2004-2007 at the end of the sample.
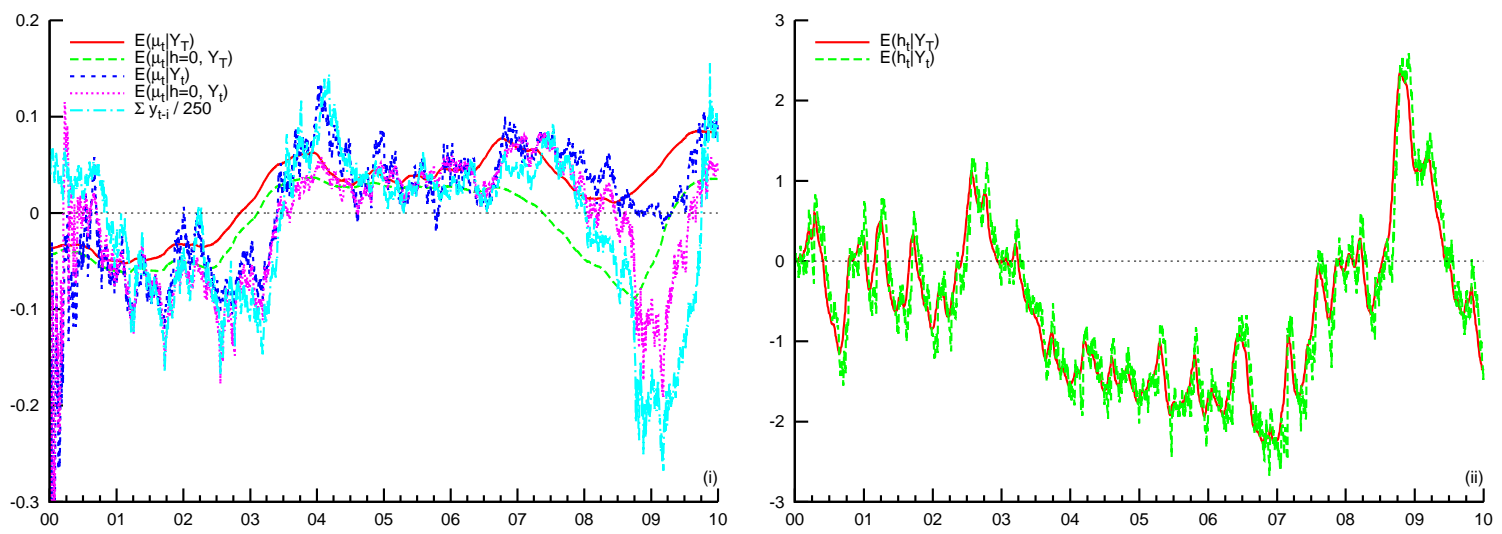

Figure 8: Comparing smoothed and filtered state estimates, for the level (panel (i)) and volatility (panel (ii)), according to the LL $(h \equiv 0)$ and LL-SV models

In Section 2.4 a particle filter for the linear state space model was introduced. Extending it to allow for time varying volatility is straightforward: Sample new volatilities $h_{t}^{(i)} \sim \operatorname{NID}\left(h_{t-1}^{(i)}, \sigma_{\nu}^{2}\right)$ in each step, and adapt for the volatility in the calculated weights. Figure 8 displays the results for the states using 10,000 particles, evaluated at the posterior mean estimates of the parameter as in Table 5 .

Panel (i) compares the smoothed level from the Gibbs sampler, $E\left(\mu_{t} \mid Y_{T}\right)$, to the smoothed level from the Gibbs sampler for the LL model, $E\left(\mu_{t} \mid h=0, Y_{T}\right)$, as well as to the filtered levels $E\left(\mu_{t} \mid Y_{t}\right)$ and $E\left(\mu_{t} \mid h=0, Y_{t}\right)$. Additionally, the average yearly return, $\sum_{i=0}^{249} y_{t-i} / 250$ is plotted. For the first part of the sample, both the particle filtered states, $E\left(\mu_{t} \mid Y_{t}\right)$ and $E\left(\mu_{t} \mid h=0, Y_{t}\right)$, and the data-based average yearly return are fairly similar in structure. It is only at the advent of the financial crisis that the estimates start to differ. As the volatility increases, the signal-to-noise ratio drops strongly, and hence after 2008 the particle filter for the LL-SV model does not adapt the state estimate much. The estimate $E\left(\mu_{t} \mid h=0, Y_{T}\right)$ is adapted much more when the constant variance LL model is used.

Panel (ii) of Figure 8 compares the smoothed and filtered volatility estimates. Both estimates agree closely, with the filtered estimate naturally being less smooth. It is however rather remarkable to see how little difference there is between the volatility extracted conditional on the full data set, or conditioning on only past data. The variance $\sigma_{\nu}^{2}$ of the volatility series is such that $h_{t}$ is estimated mostly on the basis of present information, and both past and future information are less important. For the level, in the first panel, the difference 
between the smoothed and filtered estimates is far larger. As the data itself contains little information on the expected returns, information over a longer horizon is combined. If future information is available, as in the smoothed estimate of the state, then this information can alter the estimate of the state considerably.

The particle filter could also be used to construct a likelihood estimate, which in turn could serve as input for the calculation of the marginal likelihood (see Equation (5)). Table 5 reports the values for the marginal loglikelihoods. The LL-SV model has a marginal loglikelihood which is 641 points higher than that of the LL model. According to the scheme of Kass and Raftery (1995), this log-difference of 641 is an indication that stochastic volatility is an indispensable part of the model for S\&P 500 returns.

\section{Concluding remarks}

The purpose of this article was to present methods, algorithms and implementations for analysing the Nile river flow data using $\mathrm{Ox}$ in combination with Bayesian Markov chain Monte Carlo techniques. Section 2 concludes that the Bayesian analysis is able to mimic closely the results from a classical analysis, so one could think that the Bayesian approach here is of little added value.

In Section 3 the extension towards a model with time varying variance is made. Within the Gibbs sampler, such an extension is easily implemented. From the results, some indication is found that volatility in the river flow dropped after 1916. Uncertainty about the volatility is large, as the number of observations is very limited. The marginal likelihood indicated that indeed the data does not favour a time varying volatility.

The posterior of the parameters in this case indicated multimodality of the density of $\sigma_{\varepsilon}$ and $\sigma_{\xi}$. The Bayesian approach gives a clear signal that different parameter settings could describe the data well. In a classical approach, such multimodality is often hard to recognise, and it is not always clear whether reported maximum likelihood estimates are from the global or possibly from a local maximum.

The local level plus volatility model is applied in Section 4 on returns of the S\&P500 data. The existing programs could be used with little change to extract a heavily time varying volatility for these financial index returns. For this series, extracting the expected return as measured by the local level of the model is harder, as this data is more informative on the volatility than on the level. The marginal likelihood in this case overwhelmingly favours the local level model with stochastic volatility, instead of the standard linear Gaussian unobserved components model with fixed variances.

In all these cases, an implementation in Ox was described in detail, and with short programs these extensive models could be estimated without further problems. This serves to show the flexibility of both the language and of the Bayesian MCMC approach applied here.

\section{A The Simulation Smoother}

In Durbin and Koopman (2002) a simple and efficient simulator for the states of a model in state space is proposed. It can be implemented using a few lines of code in Ox as in Listing 7 .

Listing 7: The simulation smoother in Ox

SimSmoDK (const vY, const mPhi, const mOmega, const mSigma) 
\{

decl vAlpha, mU, iT, mP, mR, mYStar, mD, ir;

$\mathrm{iT}=\operatorname{sizerc}(\mathrm{vY})$; // Generate new pseudo-data

$\mathrm{mU}=\operatorname{rann}(2,1) \sim \operatorname{choleski}(\mathrm{mOmega}) * \operatorname{rann}(2, \mathrm{iT})$;

// Don't use diffuse initialisation for recursion

$\mathrm{mP}=\operatorname{mSigma}[0][0]=-1$ ? 1 : $\operatorname{sqrt}(\mathrm{mSigma}[0][0])$;

$\mathrm{mR}=$ SsfRecursion (mU, mPhi, mOmega, mP|mSigma[1][]);

mYStar $=v Y-m R[1][1:] ; \quad$ // Smooth the difference with y

SsfMomentEst(ST_SMO, \&mD, mYStar, mPhi, mOmega, mSigma[0][]|0);

// The simulated state is the sum of pseudo-state and smoothed state

\}

return $m R[0][: i T-1]+m D[0][: i T-1]$;

Such code follows Algorithm 1 of Durbin and Koopman (2002) closely:

1. In the first step, a set of random disturbances is generated, which afterwards are run through the recursion of Equations (1)-(2) using the function SsfRecursion() from SsfPack. Note that here the initial standard deviation should be provided in the last function argument, instead of the variance in mSigma. This results in pseudo-data $y^{+}$ and $\alpha^{+}$in the matrix $\mathrm{mR}$.

2. Secondly, $y^{*}=y-y^{+}$is calculated, the difference between the true and the pseudo-data. This pseudo-data is smoothed, using SsfMomentEst(), resulting in $\hat{\alpha}$.

3. The simulated states are now the difference between the pseudo-states $\alpha^{+}$and the smoothed states $\hat{\alpha}$. This difference is returned from the function.

Here, the simulation smoother is implemented for the most simple state space model. With minor changes similar code would work as well for models with e.g. time varying variances, with intercepts, or other extensions to the basic local level model.

\section{B List of programs and routines}

The algorithms described in this article have been implemented in Ox (Doornik, 2009), making use of a few routines in SsfPack 2.2 (Koopman et al., 1999). For graphical output, the professional version of Ox is needed; alternatively, the GnuDraw package of Bos (2003) can be used.

The code for these algorithms is available at the website of the journal, together with the necessary data. The code is split into several self-contained programs, as listed in Table 6 . The routines that have been discussed in more detail, including their Ox code, are listed in Table 7 .

\section{References}

Bauwens, L., M. Lubrano, and J.-F. Richard (1999). Bayesian Inference in Dynamic Econometric Models. Advanced Texts in Econometrics. Oxford: Oxford University Press. 
Table 6: List of programs

\begin{tabular}{lllcl} 
Section & File & Data & SV & Method \\
\hline 2.2 & estnilemh.ox & Nile & - & Metropolis-Hastings \\
2.3 & estnilegb.ox & Nile & - & Gibbs with data augmentation \\
2.4 & estnilepf.ox & Nile & - & Particle filter \\
2.5 & estnileml.ox & Nile & - & Maximum likelihood \\
3 & estnilegbsv.ox & Nile & + & Gibbs with data augmentation \\
4 & estspgbsv.ox & S\&P500 & + & Gibbs with data augmentation \\
4 & estsppfsv.ox & S\&P500 & + & Particle filter \\
\hline \hline
\end{tabular}

\begin{tabular}{llll} 
& & \multicolumn{2}{c}{ Table } \\
Listing & Section & Routine & Purpose \\
\hline 1 & 2.2 & EstBayesMH & The random walk Metropolis scheme \\
2 & 2.2 & LnPosterior & Logarithm of the posterior density \\
3 & 2.3 & SimSigmaIG1 & Simulating from the IG-1 density \\
4 & 2.4 & PartFiltLL & The particle filter \\
5 & 2.4 & MargLiklLP & Marginal likelihood using Laplace \\
6 & 3.2 & SampleHAR & The single-site sampler for $h_{t} \mid h_{t-1}, h_{t+1}, y_{t}, \theta$ \\
7 & $\mathrm{~A}$ & SimSmoDK & The simulation smoother \\
\hline \hline
\end{tabular}

Bos, C. S. (2002). A comparison of marginal likelihood computation methods. In W. Härdle and B. Ronz (Eds.), COMPSTAT 2002 - Proceedings of Computational Statistics, pp. 111117.

Bos, C. S. (2003). GnuDraw. http://www.tinbergen.nl/〜cbos/gnudraw.html.

Bos, C. S. and N. Shephard (2006). Inference for adaptive time series models: Stochastic volatility and conditionally Gaussian state space form. Econometric Reviews 25(2-3), 219-244.

Cappé, O., S. J. Godsill, and E. Moulines (2007). An overview of existing methods and recent advances in sequential Monte Carlo. IEEE Proceedings 95 (5), 899-924.

Chib, S. and E. Greenberg (1995). Understanding the Metropolis-Hastings algorithm. The American Statistician 49(4), 327-335.

Commandeur, J. J. F., S. J. Koopman, and M. Ooms (2011). Statistical software for state space methods. Journal of Statistical Software. Forthcoming.

Doornik, J. A. (2009). Ox6: An Object-Oriented Matrix Programming Language. London: Timberlake Consultants Ltd.

Durbin, J. and S. J. Koopman (2001). Time Series Analysis by State Space Methods. Oxford: Oxford University Press.

Durbin, J. and S. J. Koopman (2002). A simple and efficient simulation smoother for state space time series analysis. Biometrika 89(3), 603-615. 
Geweke, J. (1992). Evaluating the accuracy of sampling-based approaches to the calculation of posterior moments. In J.-M. Bernardo, J. O. Berger, A. P. Dawid, and A. F. M. Smith (Eds.), Bayesian Statistics 4: Proceedings of the Fourth Valencia International Meeting, pp. 169-193. Oxford: Clarendon Press.

Harvey, A. C. (1989). Forecasting, Structural Time Series Models and the Kalman Filter. Cambridge: Cambridge University Press.

Jeffreys, H. (1961). Theory of Probability (3rd ed.). Oxford, U.K.: Oxford University Press.

Kass, R. E. and A. E. Raftery (1995). Bayes factors. Journal of the American Statistical Association 90(430), 773-795.

Kass, R. E., L. Tierney, and J. B. Kadane (1990). The validity of posterior expansions based on Laplace's method. In S. Geisser (Ed.), Bayesian and Likelihood Methods in Statistics and Econometrics: Essays in Honor of George A. Barnard, pp. 473-488. North Holland, Amsterdam.

Kim, S., N. Shephard, and S. Chib (1998). Stochastic volatility: Likelihood inference and comparison with ARCH models. Review of Economic Studies 65(3), 361-393.

Koopman, S. J., N. Shephard, and J. A. Doornik (1999). Statistical algorithms for models in state space using SsfPack 2.2. Econometrics Journal 2(1), 107-160.

Koopman, S. J., N. Shephard, and J. A. Doornik (2008). SsfPack 3.0: Statistical Algorithms for Models in State Space Models. London: Timberlake Consultants Ltd.

Metropolis, N., A. W. Rosenbluth, M. N. Rosenbluth, A. H. Teller, and E. Teller (1953). Equations of state calculations by fast computing machines. Journal of Chemical Physics 21, $1087-1091$.

Pitt, M. K. and N. Shephard (1999). Filtering via simulation: Auxiliary particle filters. Journal of the American Statistical Association 94(446), 590-599.

Smith, A. F. M. and A. E. Gelfand (1992, May). Bayesian statistics without tears: A samplingresampling perspective. The American Statistician 46(2), 84-88. 